\title{
Evaluación de competencias de las regiones para el desarrollo de un clúster útil a la cadena productiva de pasifloras en Colombia
}

Assessment of Regional Competencies for the Development of a Cluster Useful to the Passion Flower Production Chain in Colombia

Avaliação de competências das regiões para o desenvolvimento de um cluster útil à cadeia produtiva de passifloras na Colômbia

\author{
Leila N. Ramírez \\ Ángela M. Otálvaro ${ }^{* *}$
}

Fecha de recibido: 18 de julio de 2014

Fecha de aprobado: 9 de diciembre de 2014

Doi: dx.doi.org/10.12804/rev.univ.empresa.28.2015.03

Para citar este artículo: Ramírez, L. N., \& Otálvaro, A. M. (2015). Evaluación de competencias de las regiones para el desarrollo de un clúster útil a la cadena productiva de pasifloras en Colombia. Universidad \& Empresa, 17(28), 69-85. Doi: dx.doi.org/10.12804/rev.univ.empresa.28.2015.03

Estudiante del doctorado en Ingeniería de la Pontificia Universidad Javeriana (Bogotá, Colombia), Magíster en Ingeniería Industrial de la Universidad de los Andes (Bogotá, Colombia), Ingeniera Industrial de la Universidad Libre (Bogotá, Colombia). Profesora e Investigadora del Grupo Intecom CINDES de la Facultad de Ingeniería de la Universidad Libre (Bogotá, Colombia). Correo electrónico: leylan.ramirezc@unilibrebog.edu.co

** Doctora y Magíster en Ingeniería Química e Ingeniera Química de la Universidad Nacional de Colombia (Bogotá, Colombia). Profesora Asociada de la Facultad de Ingeniería de la Universidad de la Salle (Bogotá, Colombia). Integrante Grupo Ciencia, tecnología e ingeniería en el procesamiento de alimentos. Correo electrónico: amotalvaro@unisalle.edu.co 


\section{RESUMEN}

En Colombia, el Ministerio de Agricultura y Desarrollo Rural (MADR) ha definido las cadenas productivas agrícolas con potencial para alcanzar la prosperidad regional y se encuentra impulsando iniciativas, entre las que está la creación de clústeres, con el fin de lograr ese objetivo. Esta investigación busca hacer explícita la metodología utilizada para definir las capacidades competitivas de las diferentes regiones productoras de pasifloras (frutas de carácter exótico que tiene un alto potencial de desarrollo comercial). Se trata de establecer la mejor ubicación dentro de las regiones potenciales para el clúster en esta cadena en Colombia. Se emplea una matriz de evaluación basada en el diamante de competitividad de Porter. Esto permitió establecer que el Huila es la región que alcanza el mayor nivel competitivo para el desarrollo de este tipo de agrupaciones.

Palabras clave: cadenas productivas, clúster, Colombia, competitividad, pasifloras.

\section{ABSTRACT}

In Colombia, the Ministry of Agriculture and Rural Development (MARD) has defined agricultural production chains with potential to achieve regional prosperity and is encouraging initiatives, among which is the creation of clusters to assist in at attaining this objective. This research seeks to make explicit the methodology used to clearly define the competitive capacities of the different regions where passion flowers (exotic fruits with high commercial development potential) are produced. It involves establishment of the best location within the regions of Colombia with potential for the cluster in this chain. An assessment matrix based on Porter's competitiveness diamond is applied. This has allowed Huila to be established as the region with the highest level of competitiveness for the development of this type of groups.

Key words: Production chains, cluster, Colombia, competitiveness, passionflowers.

\section{RESUMO}

Na Colômbia, o Ministério da Agricultura e Desenvolvimento Rural (MADR) tem definido as cadeias produtivas agrícolas com potencial para alcançar a prosperidade regional e encontra-se impulsando iniciativas, entre as que está a criação de clusters, com o fim de conseguir esse objetivo. Esta pesquisa busca fazer explícita a metodologia utilizada para definir as capacidades competitivas das diferentes regiões produtoras de passifloras (frutas de carácter exótico que tem um alto potencial de desenvolvimento comercial). Trata-se de estabelecer a melhor localização dentro das regiões potenciais para o cluster nesta cadeia na Colômbia. Se utiliza uma matriz de avaliação baseada no diamante de competitividade de Porter. Isto permitiu estabelecer que Huila é a região que alcança o maior nível competitivo para o desenvolvimento deste tipo de agrupações.

Palavras-chave: cadeias produtivas, cluster, Colômbia, competitividade, passifloras. 


\section{INTRODUCCIÓN}

A través del desarrollo de la historia económica y empresarial se han gestado agrupaciones o conglomerados empresariales dentro de entornos específicos, buscando sinergias $\mathrm{y}$ ventajas competitivas que dinamicen los sectores productivos. Esto ha contribuido a generar discusiones y teorías relativas al emprendimiento y la sostenibilidad, así como múltiples modelos de crecimiento económico para los países.

En relación con esto, por ejemplo, se encuentran los estudios sobre los clúster, cuyo origen teórico es atribuido a los trabajos desarrollados Marshall (1890/2005). En su obra se definen los distritos industriales como agrupaciones desectoresespecializados en una localidad específica, cuyo establecimiento genera externalidades positivas para las empresas y diversas ventajas como la especialización de la mano de obra, el ahorro de costos vinculados a desplazamientos y la generación de un mercado de trabajo constante, entre otras.

Aportando a esta temática, autores como Porter (1990) han definido los clústeres como agrupaciones de empresas e instituciones asociadas geográficamente, que se encuentran dentro de un sector específico y que están interconectadas por prácticas comunes y complementarias, grupos de organizaciones cuyo éxito depende de manera importante del buen manejo y la incorporación amplia del factor tecnológico. En el mismo sentido, Rosenfeld (1995) define un clúster como una aglomeración específica, delimitada en un espacio determinado por un número de compañías parecidas, que se vinculan para ser más competitivas como grupo. Esto difiere en alguna medida de lo planteado por Czamanski y Ablas (1979), para quienes la ubicación no es un factor determinante para estas agrupaciones, ya que su lazo principal está definido más bien por un constante flujo de bienes y servicios.

En el desarrollo de los clústeres inciden factores como las políticas gubernamentales y el entorno socioeconómico. Redman (1994) resalta el importante papel que tienen las instituciones como un factor de la competitividad. Estas denotan factores de desarrollo esenciales como la infraestructura, el capital humano y la innovación. Teniendo en cuenta esto, Jacobs y Deman (1996), por su parte, identifican otros factores relevantes, que coinciden en buena medida con mencionados por Porter, como la agrupación geográfica, el uso de tecnologías homogéneas, la cooperación entre las empresas y la actividad de un ente regulador y dinamizador para los clústeres. 
A medida que las definiciones y teorías sobre las agrupaciones empresariales han evolucionado se han vuelto cada vez más compactas (Ketels, 2006). Rosenfeld (1997), por ejemplo, añade a lo indicado antes que el tamaño de los clústeres, su importancia relativa a nivel local, los productos que se ofertan y los servicios que se utilizan también son factores a tener en cuenta para su consolidación.

Partiendo de estos conceptos, gobiernos como el de Dinamarca, han impulsado diversos sectores, entre ellos el agrícola, a través de la estrategia clústeres. A través de esto, se han logrado resultados importantes en el desarrollo regional, como la integración de forma natural de las cadenas de valor por medio de la cooperación. Así, la región del triángulo sur de Dinamarca, en particular, cubre las necesidades de ochenta millones de consumidores, empleando entre 7000 y 8000 personas en el sector, a través de 120 compañías relacionadas con el negocio. Esto ha permitido posicionar a la región como la más importante productora de alimentos de Dinamarca, facilitando así el desarrollando nuevos negocios con dinámicas similares (Europe innova, 2008).

La notoria relevancia del desarrollo regional para los Estados, como base para lograr la competitividad en un mercado global cada vez más exigente (Corrales, 2007), hace necesario que las naciones estén dispuestas a considerar estos planteamientos dentro de sus políticas. Así, el Ministerio de Agricultura y Desarrollo Rural (MADR) de Colombia, ha generado iniciativas como la creación de los Consejos Nacionales de las Cadenas Productivas. Esto con el fin de permitir que las cadenas productivas ubicadas a lo largo de las regiones se conviertan en el eje articulador de un proceso agrícola más dinámico, apoyando la concertación, difusión y ejecución entre los actores concernidos, como base para incrementar la competitividad.

Una de las cadenas productivas definidas como estratégica para Colombia es la de las Pasifloras. Esta incluye frutas como el maracuyá, la granadilla, la gulupa, la chulupa, la badea y la curuba, las cuales han adquirido importancia, en particular, por su potencial de exportación (Lobo \& Medina, 2009).

Según el MADR (2012), Colombia es uno de los principales productores de pasifloras. La granadilla, por citar solo un ejemplo, alcanzó la importante producción de 43885 toneladas en 2007 y de 52305 toneladas en 2008. 
Los cultivos de pasifloras se encuentran distribuidos en este país principalmente en las siguientes regiones (Consejo Nacional de Pasifloras, 2012):

- Región de Occidente, integrada por los departamentos de Valle del Cauca, Cauca y Nariño.

- Región Tolima-Huila, compuesta por estos dos departamentos.

- Región Central, constituida por los departamentos de Cundinamarca, Boyacá y Meta.

- Región de Antioquía y el Eje Cafetero, conformada por los departamentos de Caldas, Quindío, Risaralda y Antioquia.

Para que esta cadena logre el nivel de competitividad esperado (Areiza, 1999), a través de recursos asignados por el MADR, se busca generar una infraestructura organizacional y operativa que permita articular a instituciones gubernamentales como las Secretarias de Agricultura, el Consejo de la Cadena Productiva de las Pasifloras, el Servicio Nacional de Aprendizaje (SENA), la Corporación Colombiana de Investigación Agropecuaria (Corpoica) y el Instituto Colombiano Agropecuario (ICA) con el sector industrial, integrando así a la agroindustria con los productores y las instituciones académicas y de investigación, por medio de la metodología de clústeres.
Esto, justamente, genera la necesidad de evaluar las regiones en cuanto a sus ventajas competitivas, con el fin de establecer la ubicación geográfica más adecuada para el clúster, algo que, de acuerdo con la literatura antes mencionada, es importante para su desarrollo.

\section{CLÚSTERES AGRÍCOLAS EN COLOMBIA}

En Colombia se ha desarrollado una serie de estudios para determinar la caracterización de clústeres agrícolas con base en la metodología de Porter. Esta sugiere determinar las actividades económicas interrelacionadas y la concentración actual de empleo de dichas actividades para considerar la ubicación de los mismos. Se han realizado también múltiples agendas de investigación con el fin de generar estrategias y de apropiar el conocimiento a través de un sistema de inteligencia tecnológica. Esto como apoyo a la toma de decisiones de priorización en materia de demandas de investigación y de desarrollo de diferentes cadenas productivas en el país.

Como resultado de estas actividades se destaca, por ejemplo, un estudio realizado por el Consejo Privado de Competitividad de Bogotá (2010). Algunos de sus principales hallazgos se presentan en la tabla 1 . 
Tabla 1. Clústeres identificados en Colombia

\begin{tabular}{|c|c|c|c|}
\hline Producto & \multicolumn{2}{|c|}{ Satisfacción } & Clúster \\
\hline Lácteos & \multirow{7}{*}{ 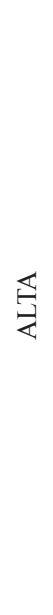 } & 18.465 & $\begin{array}{l}\text { Cauca (en desarrollo), Nariño (en desarrollo), César } \\
\text { (potencial). }\end{array}$ \\
\hline $\begin{array}{l}\text { Cacao, chocolate, } \\
\text { confitería }\end{array}$ & & 17.078 & $\begin{array}{l}\text { Antioquia (establecido), Cauca (en desarrollo), Huila } \\
\text { (potencial). }\end{array}$ \\
\hline Azúcar & & 16.804 & Valle (establecido), César (potencial), Risaralda (potencial) \\
\hline Carne y derivados & & 15.057 & $\begin{array}{l}\text { Risaralda (en desarrollo), Santander (en desarrollo), Bolívar } \\
\text { (en desarrollo), Caquetá (potencial), César (potencial), } \\
\text { Guaviare (potencial), Putumayo (potencial). }\end{array}$ \\
\hline Pescado y derivados & & 13.627 & Bolívar (establecido), Atlántico (en desarrollo). \\
\hline $\begin{array}{l}\text { Molinería, panadería } \\
\text { y concentrados }\end{array}$ & & 12.950 & $\begin{array}{l}\text { Huila (en desarrollo), Meta (potencial), Tolima (potencial), } \\
\text { Córdoba (potencial), César (potencial). }\end{array}$ \\
\hline $\begin{array}{l}\text { Compuestos de frutas } \\
\text { y hortalizas }\end{array}$ & & 12.005 & Antioquia (establecido). \\
\hline Bebidas alcohólicas & \multirow{3}{*}{ 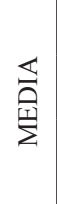 } & 10.911 & Bogotá-Cundinamarca (establecido), Bolívar (potencial) \\
\hline Productos de tabaco & & 9.627 & Antioquia (establecido) \\
\hline Aceites y grasas & & 7.530 & $\begin{array}{l}\text { Santander (establecido), Atlántico (en desarrollo), } \\
\text { Magdalena (en desarrollo), Córdoba (potencial). }\end{array}$ \\
\hline Panela & \multirow{3}{*}{ 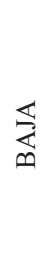 } & 5.830 & Valle (establecido). \\
\hline Café y derivados & & 4.896 & $\begin{array}{l}\text { Caldas (en desarrollo), Cauca (establecido), Nariño (en } \\
\text { desarrollo), Tolima (en desarrollo), Córdoba (en desarrollo), } \\
\text { Magdalena (potencial) }\end{array}$ \\
\hline $\begin{array}{l}\text { Bebidas no } \\
\text { alcohólicas }\end{array}$ & & 4.461 & $\begin{array}{l}\text { Bolívar (establecido), Boyacá (en desarrollo), Tolima } \\
\text { (potencial), Cauca (potencial), Córdoba (potencial). }\end{array}$ \\
\hline
\end{tabular}

Fuente: Mapa de clúster agroindustriales en Colombia, Consejo Privado de Competitividad de Bogotá (2010).

En la tabla 1 se observa que se han identificado varios clústeres potenciales cuyo desarrollo es aún incipiente. Con base en esto, es posible indicar que en Colombia este tipo de iniciativas se encuentra todavía en un estado inmaduro y que, en consecuencia, tiene importantes posibilidades para evolucionar hacia el futuro. 
2. VENTAJAS DEL DESARROLLO DE CLÚSTERES PARA EL SECTOR AGRÍCOLA

El desarrollo de diversos negocios se centra en sus estrategias de desarrollo (Pettigrew, Thomas, \& Whittington, 2010; Avendaño, 2008). Dentro de estas se encuentran, entre otras, la implementación de tecnologías de información, servicios y procesos y la incorporación y desarrollo de diferentes tipos de innovaciones (Icki, Leguizamón, Metzger \& Flores, 2009). Estas estrategias permiten el desarrollo económico de componentes específicos dentro de las cadenas productivas y con ellas es posible potencializarlas (McCormick, 2005), generando sinergias entre los actores involucrados para alcanzar mayor y mejores resultados conjuntos (Karlsson, 2008; Håkanson, 2005).

La cooperación trae consigo beneficios individuales para las organizaciones participantes (Brandenburger, \& Nalebuff, 1996; Federal Ministry of Economics and Technology, 2009), como laidentificación de fortalezas individuales, la concentración de esfuerzos, la especialización en temas específicos, la adición de competencias necesarias, la construcción de un valioso know how, el mejoramiento del estado de la información con la que se cuenta, el uso común de conocimientos y recursos, el aumento de la productividad a partir de la rápida difusión de la tecnología, las facilidades en la comercialización a través de canales de venta conjuntos, el desarrollo de mercados transparentes, la obtención de costos relativamente bajos en el mercado, la apertura de nuevos espacios para comerciar, así como la adquisición de nuevos clientes y el desarrollo de estrategias para alcanzar rendimientos conjuntos.

Por lo anterior, y por los beneficios que pueden generar para el desarrollo regional y para la economía en general a mediano y largo plazo, existe un amplio interés en configurar estrategias tipo clúster para el sector agrícola. Sin embargo, es importante mencionar que para que estos puedan operar apropiadamente se necesita una adecuada infraestructura de movilidad que sea sostenible y que parta de las cadenas de suministro (Ketels, 2007).

\section{METODOLOGÍA}

La estrategia desarrollada para definir la ubicación geográfica adecuada para la ubicación del clúster de la cadena de las pasifloras en Colombia, parte del estudio del modelo del diamante de Porter. Este se presenta en la figura 1 . 
Figura 1. Modelo del diamante de Porter

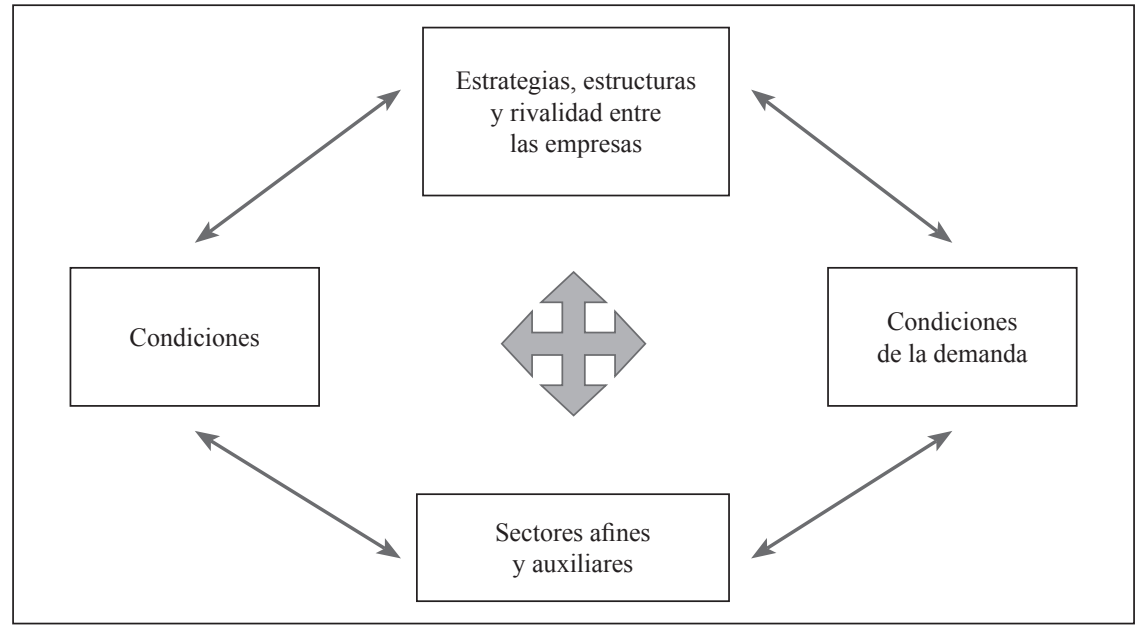

Fuente: (Porter, 1990, p. 78).

En la figura 1 se observa que los clústeres superan en su esencia la simple interacción empresarial de compra y venta de productos. Estos dan lugar también a sinergias con otros agentes como los centros de conocimiento e innovación, el Estado, entre otros (Feldman, Francis \& Bercovitz, 2005). Así, construyen ventajas integrales y se promueve la creación de valor, haciendo uso de un modelo que, a través del apoyo mutuo, promueve el incremento de la competitividad (Menzel \& Fornahl, 2010).

Los beneficios esperados de la implementación de un clúster constituyen elementos estratégicos importantes para lograr un desempeño exitoso de los sectores (Cassiolato \& Lastres, 2000). Este aporta a su con- solidación en el mercado local y global, a partir de redes de colaboración que se fortalecen por medio de esta iniciativa (Gil Bolivar, 2011).

Considerando lo anterior, se decidió construir una matriz en donde se involucraran determinados criterios que permitieran evaluar las competencias de las principales regiones productoras de pasifloras en Colombia. Esto con el fin de poder seleccionar adecuadamente a una de ellas como la ubicación más apropiada para el clúster.

Con base en el modelo del diamante de Porter, se hizo la siguiente particularización de acuerdo con la aplicación específica que se está desarrollando actualmente: 


\subsection{Condiciones de los factores (factores productivos)}

El estado de los factores productivos establece elementos asociados a la cualificación de la mano de obra, la infraestructura, la tecnología y el uso sostenible de los recursos. El objetivo principal de evaluar esta categoría es la necesidad de identificar las actividades en las que se debe ser fuerte para lograr una alta productividad y cumplir con apuestas de valor específicas.

Este criterio busca establecer la relación de la oferta con respecto a la demanda del mercado, permitiendo visualizar la capacidad de las unidades productivas de satisfacer las demandas. Los elementos seleccionados para ser evaluados en este punto son: área disponible de cultivo, número de productores, número de distribuidores, número de transformadores, disponibilidad de los recursos, nivel de remuneración de la fuerza trabajo, infraestructura de transporte, acceso a sistemas de riego, acceso a sistemas financieros, acceso a nuevas tecnologías, activos fijos obsoletos, niveles de inversión de capital, disponibilidad de información y vulnerabilidad a plagas. En la tabla 2, se hace un resumen de los factores productivos considerados.

Tabla 2. Definición de algunos elementos para evaluar los criterios productivos

\begin{tabular}{|l|l|l|}
\hline Elemento evaluado & \multicolumn{1}{|c|}{ Definición del factor } & \multicolumn{1}{|c|}{$\begin{array}{l}\text { Unidad utilizada } \\
\text { para su evaluación }\end{array}$} \\
\hline $\begin{array}{l}\text { Área disponible } \\
\text { para el cultivo }\end{array}$ & $\begin{array}{l}\text { Extensión de tierra apta para la producción de } \\
\text { pasifloras en la región, acotada por una superficie } \\
\text { preestablecida. }\end{array}$ & Hectáreas \\
\hline $\begin{array}{l}\text { Número de } \\
\text { productores }\end{array}$ & $\begin{array}{l}\text { Número de personas con responsabilidad económica } \\
\text { que organizan y financian cultivos de pasifloras. }\end{array}$ & $\begin{array}{l}\text { Número de } \\
\text { personas }\end{array}$ \\
\hline $\begin{array}{l}\text { Número de } \\
\text { distribuidores }\end{array}$ & $\begin{array}{l}\text { Número de personas naturales o jurídicas que se } \\
\text { encargan de comercializar como mayoristas o } \\
\text { minoristas los productos de esta cadena, bien sea a } \\
\text { otros mayoristas, a minoristas, o al consumidor final. }\end{array}$ & $\begin{array}{l}\text { Número de } \\
\text { distribuidores }\end{array}$ \\
\hline $\begin{array}{l}\text { Número de } \\
\text { transformadores }\end{array}$ & $\begin{array}{l}\text { Número de personas naturales o jurídicas que reciben } \\
\text { las pasifloras como materia prima y las transforman } \\
\text { en subproductos. }\end{array}$ & $\begin{array}{l}\text { Número de } \\
\text { transformadores }\end{array}$ \\
\hline $\begin{array}{l}\text { Disponibilidad de } \\
\text { recursos naturales } \\
\text { (agua) }\end{array}$ & $\begin{array}{l}\text { Número de fuentes de agua con posibilidades de ser } \\
\text { utilizadas para apoyar el desarrollo de los cultivos } \\
\text { (ríos y quebradas naturales). }\end{array}$ & $\begin{array}{l}\text { Número de ríos o } \\
\text { quebradas }\end{array}$ \\
\hline
\end{tabular}

Fuente: elaboración propia. 


\subsection{Condiciones de la demanda}

Este aspecto permite establecer las condiciones vigentes de oferta de productos innovadores para definir los canales de distribución actuales y proporcionar una orientación acerca del posicionamiento de los productos agrícolas de pasifloras en el mercado local e internacional. Los elementos definidos para este criterio son: productos desarrollados, innovación en la producción, volumen de producción (en fresco), volumen de producción (producto transformado), innovación e inversión privada en el sector, caracterización de los clientes (nivel de sofisticación de los mismos), tipo de mercado (local/nacional/internacional) y volumen en el mercado (distribuido según nivel). Como en el caso anterior, se definió cada factor y se asignó una unidad para su cuantificación.

\subsection{Estrategia, estructura y rivalidad}

En este punto se evalúa la capacidad de estructuración de las competencias frente a la globalización de los mercados y el desarrollo de estrategias de gestión logística para alcanzar la sostenibilidad del agronegocio en el largo plazo. Los elementos que componen este criterio son: orientación a la exportación, restricción a la fase productiva, fijación de precios en mercados locales, planes de expansión del mercado a largo plazo, presencia de mercados internos desordenados y gestión para la logística internacional. Una vez más, como en los casos anteriores, se definió cada factor y se asignó una unidad para su cuantificación.

\subsection{Industrias de apoyo y relacionadas}

Dentro de las características diferenciadoras de la estructura de clúster se resalta la importancia de las relaciones de colaboración con miembros de la cadena de valor y los interesados externos a la operación industrial, como una herramienta para potencializar el desarrollo.

Dentro de estos interesados externos se encuentran centros de investigación e instituciones de carácter público y privado, entre otros. La existencia de ese tipo de interrelaciones se puede evidenciar a través de esta categoría. Los elementos evaluados aquí son: vínculos entre los miembros de la cadena (agremiaciones, alianzas, etc.), número de organizaciones proveedoras de insumos ubicadas en la región, número de organizaciones proveedoras de servicios de apoyo ubicadas en la región, especialización del recurso humano, servicios de logística a disposición de la cadena e instrumentos de califi- 
cación. Nuevamente se definió cada factor y se asignó una unidad para su cuantificación.

La definición de los elementos contemplados en cada criterio fue desarrollada, en lo fundamental, a partir de 1) la lectura de otros trabajos en los que se relacionan experiencias similares (Czamanski \& Ablas, 1979; Amórtegui \& Carvajal, 2006; Europe innova, 2008; Federal Ministry of Economics and Technology, 2009; Clarkson, M.,Clarkson, M., Fink, M., \& Kraus, S., 2007; Rodríguez \& Naranjo, 2008) y 2) la consideración de los aspectos específicos asociados al contexto de aplicación considerado, es decir, la cadena productiva de las pasifloras (MADR, 2011).

La calificación de cada elemento dentro de la matriz se realizó a partir de una escala compuesta por tres niveles que permiten definir, para cada uno de ellos, el estado de madurez alcanzado por las diferentes regiones evaluadas, frente a su nivel de competencias en las diversas categorías.

En la tabla 3, a manera de ilustración, se pueden observar las escalas de evaluación definidas para tres de los elementos considerados en el criterio factores de la demanda.

Tabla 3. Ejemplo de las escalas de evaluación utilizadas para algunos elementos

\begin{tabular}{|l|l|}
\hline \multicolumn{1}{|c|}{ Elementos } & \multicolumn{1}{c|}{ Escala de evaluación } \\
\hline Area disponible para el cultivo & $\begin{array}{l}\text { Nivel Bajo: menos de } 100 \mathrm{H}, \\
\text { Nivel Medio: entre } 100 \mathrm{y} 1000 \mathrm{H} \text { y } \\
\text { Nivel Alto: más de } 1000 \mathrm{H}\end{array}$ \\
\hline Número de productores & $\begin{array}{l}\text { Nivel Bajo: menos de } 50, \\
\text { Nivel Medio: entre } 50 \text { y } 100 \\
\text { Nivel Alto: más de } 100\end{array}$ \\
\hline Número de distribuidores & $\begin{array}{l}\text { Nivel Bajo: menos de } 50, \\
\text { Nivel Medio: entre } 50 \text { y } 100 \\
\text { Nivel Alto: más de } 100\end{array}$ \\
\hline
\end{tabular}

Fuente: elaboración propia.

Las ponderaciones para cada una de las escalas de evaluación se definieron de acuerdo a la importancia del valor frente a la competitividad. Para la evaluación de cada una de las regiones se estableció el método Delphi (Wroblewski \& Leitner, 2009), basado en los criterios de matriz del modelo de competitividad de Porter. 
El grupo de expertos estaba compuesto por algunos secretarios de agricultura de las regiones productoras y líderes de las asociaciones de productores. De ese modo, por medio de la Secretaría Técnica de la Cadena de Pasifloras se hizo llegar a cada una de las Secretarías de Agricultura regionales que potencializa cultivos de pasifloras el formato con la matriz y la escala de evaluación para que se asignara la calificación a cada elemento. Para el estudio se contó con la participación de cinco de estas, que cuentan actualmente con áreas importantes de cultivo.

Con la información obtenida de los expertos, se procedió a calificar cada elemento a través de una relación cuantitativa en una escala de 1 a 3 , otorgando una calificación de 1 al nivel más bajo, 2 al nivel medio y 3 al nivel alto. Se asumió que todos los elementos eran igualmente importantes y se procedió a realizar la suma de las calificaciones por elemento, así como la suma total de estas para tener la calificación global de cada región. De este modo, la región que tuviera una mayor calificación global sería considerada como la más prometedora para la ubicación del clúster.

Cada uno de los elementos evaluados, así como los niveles empelados para su calificación por medio del instrumento generado, se validaron también con expertos (Bogner, Littig, \& Menz, 2009) para disminuir el error en la aplicación de la prueba. El panel estaba conformado por profesores universitarios de las Universidades Libre, La Salle y Javeriana, todos con experiencia en investigación en áreas relacionadas con el sector agrícola. Finalmente, además, la información se validó con la directora del Consejo de Pasifloras.

\section{RESULTADOS}

A pesar de que se hizo una convocatoria para la aplicación de la prueba, no todas las regiones productoras de pasifloras participaron. En algunos casos esta situación es probablemente el fruto del desarrollo incipiente del agronegocio o de la desorganización en las estructuras organizacionales al interior de las regiones.

Como resultado, se obtuvo información de las regiones de Urrao, Abriaquí, Boyacá, Huila y Antioquia, y quedaron por fuera de la evaluación las regiones de Tolima y Meta. Las regiones que se analizan en el estudio conforman 3500 productores beneficiados de estos cultivos, del total nacional de 6500 productores (MADR, 2012) y así, dada la aglomeración de productores en las regiones que aportaron la información, se puede definir que las posibilidades de clúster son notorias. Dentro 
de las regiones participantes, Urrao y Abriaquí se destacaron al participar gracias a su fortaleza en asociatividad y a su experiencia en el cultivo de pasifloras.

En la figura 2 se condensan los resultados de la aplicación de la matriz a las regiones que respondieron a su diligenciamiento. Sobre cada eje se señala un criterio de los establecidos por Porter en su modelo de diamante. La escala corresponde a la valoración cuantitativa señalada como 1 nivel bajo, 2 nivel medio y 3 nivel alto de competitividad regional.

Figura 2. Comparación de la evaluación de competencias de cada una de las regiones

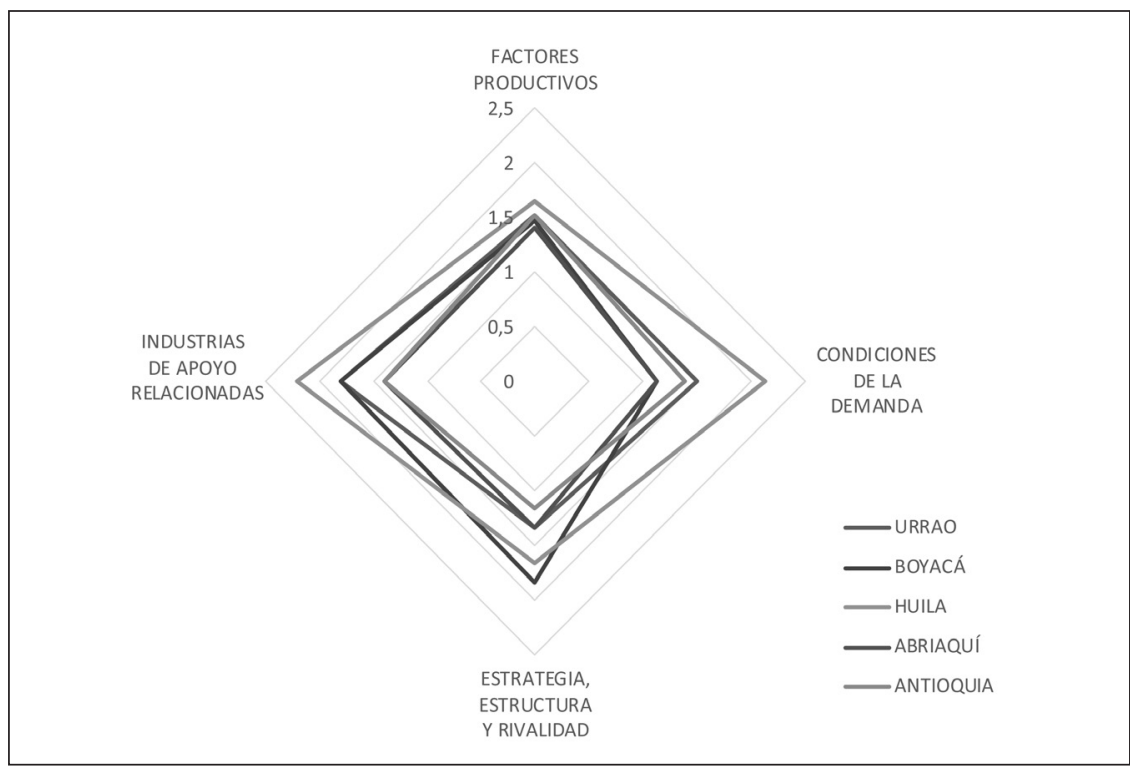

Fuente: elaboración propia.

En cuanto a la competitividad de las regiones en donde se cultivan pasifloras, para el establecimiento de un clúster, se aprecia que la más preparada para evolucionar a una estructura organizacional madura es la del Huila. Esta presenta las mejores calificaciones en casi todos los criterios y solo es superada levemente por la región de Boyacá, la cual podría ser tal vez la siguiente en la lista para la selección de la ubicación del clúster.

El posicionamiento obtenido por la región del Huila está estrechamente relacionado con su estatus de mayor productor. Esto le ha permitido 
llevar a cabo diferentes estrategias para incrementar su competitividad, como la realización de actividades de investigación (p. ej., en la Corporación Centro de Investigación para la Gestión Tecnológica de las Pasifloras del Departamento del Huila, CEPASS), el desarrollo de tecnologías para la transformación del producto, la apertura de mercados en el exterior por parte de los productores, el esfuerzo por integrar buenas prácticas agrícolas y la generación de una mayor agremiación, entre otros.

\section{CONCLUSIONES}

En esta investigación se generaron elementos para evaluar las categorías propuestas por Porter en su modelo de diamante, con el fin de establecer la competitividad de las regiones en Colombia para llegar a ser seleccionadas como punto de ubicación del clúster de pasifloras en este país. Se espera que, con base en este estudio, otros investigadores puedan también aplicar la herramienta de evaluación para determinar las brechas y la capacidad competitiva de la industria en diferentes sectores.

Los criterios considerados en la evaluación pueden emplearse en la toma de decisiones en lo referente a la competitividad de las regiones, un aspecto que debe estar establecido dentro de la metodología de clústeres. Es- tos fueron adaptados para evaluar la potencialidad frente a la estructura organizacional, pero se podrían considerar también para otras experiencias similares en sectores agrícolas diferentes.

La escala de medición puede ser considerada igualmente, con una argumentación aún más formal, como las escalas de Likert, para disminuir la subjetividad de los expertos. Sin embargo, la herramienta confirma actualmente la capacidad de la región del Huila para responder a mercados más exigentes, en comparación con otras regiones del país que producen las mismas frutas tropicales.

Este ejercicio permitió evidenciar que existen brechas que obstaculizan el desarrollo de esta cadena y, a pesar de que se evidencian esfuerzos importantes de los actores de la misma para mejorar, se aprecia que estos son aislados. Esto demuestra la necesidad de mejorar el liderazgo del Estado en la conformación de estructuras para potencializar el desarrollo regional.

\section{REFERENCIAS}

Avendaño R., B. D. (2008). Globalización y competitividad en el sector hortofrutícola: México, el gran perdedor. El Cotidiano, 23(147), 91-98. 
Amórtegui,L.,\&Carvajal,G.(2006). Propuesta de una iniciativa de Clúster. El caso de Clúster del Carbón del norte de Cundinamarca, CCNC [Archivos de economía, documento No. 314]. Bogotá: Departamento Nacional de Planeación. Recuperado de https:// colaboracion.dnp.gov.co/CDT/ Estudios\%20Econmicos/314.pdf Areiza, C. (1999). Algunas reflexiones para comercializar perecederos el caso de las frutas. Estudios gerenciales, 15(72), 13-22.

Bogner, A., Littig, B., \& Menz, W. (Eds.). (2009). Interviewing experts. Basingstoke - Nueva York: Palgrave Macmillan.

Brandenburger, A., \& Nalebuff, B. (1996). Co-opetition. Nueva York: Doubleday.

Concejo Nacional de Pasifloras. (2012). Acuerdo de Competitividad de la Cadena Productiva de Pasifloras en Colombia. Bogotá, D.C.: Ministerio de Agricultura y Desarrollo Rural.

Corrales, S. (2007). Importancia del clúster en el desarrollo regional actual. Frontera Norte, 19(37), 173-201

Clarkson, M., Clarkson, M., Fink, M., \& Kraus, S. (2007). Industrial clusters as a factor for innovative drive - in regions of transformation and structural change: A comparative analysis of East Germany and Poland.
Journal for East European Management Studies, 12(4), pp.340364.

Cassiolato, J. E., \& Lastres, H. M. M. (2000). Local systems of innovation in Mercosur countries. Industry and Innovation, 7(1), 33-53.

Czamanski, S., \& Ablas, L. A. d. Q. (1979). Identification of industrial clusters and complexes: A comparison of methods and findings. Urban Studies, 16(1), 61-80.

Europe INNOVA. (22-24 de octubre de 2008). "Case studies of clustering efforts in Europe: Analysis of their potential for promoting innovation and competitiveness". Europe innova conference 2008. Second Europe INNOVA conference, Lyon, France.

Federal Ministry of Economics and Technology. (2009). Cluster Management Excellence. Berlín: Federal Ministry of Economics.

Feldman, M., Francis, J., \& Bercovitz, J. (2005). Creating a cluster while building a firm: Entrepreneurs and the formation of industrial clusters. Regional Studies, 39(1), 129-141.

Gil Bolívar, F. (2011). Algunos interrogantes en torno al desarrollo de los clusters. Revista Ciencias Estratégicas, 19(26), 281-293.

Håkanson,L.(2005). Epistemic communities and cluster dynamics: 
On the role of knowledge in industrial districts. Industry and Innovation, 12(4), 433-463.

Ickis, J. C., Leguizamón, F. A., Metzger, M., \& Flores, J. (2009). La agroindustria: Campo fértil para los negocios inclusivos. Academia. Revista Latinoamericana de Administración, 43, 107-124. Jacobs, D., \& De Man, A.-P. (1996). Clusters, industrial policy and firm strategy. Technology Analysis \& Strategic Management, 8(4), 425-438.

Karlsson,C.(Ed.).(2008). Handbook of research on cluster theory. Cheltenham - Northampton: Edward Elgar.

Ketels, C. M. (2006). Michael porter's competitiveness framework -recent learnings and new research priorities. Journal of Industry, Competition and Trade, 6(2), 115-136

Ketels, C. (2007). The role of clusters in the Chemical Industry. Harvard Business School, EPCA.

Lobo, M., \& Medina, C. (2009). Recurso genético de pasifloráceas en Colombia. Bogotá: Sociedad Colombiana de Ciencias Hortícolas - Ministerio de Agricultura y Desarrollo Rural Colombia.

Marshall, A. (1890/2005). Principios de economía. Madrid: Síntesis. Menzel, M.-P., \& Fornahl, D. (2010). Cluster life cycles-dimensions and rationales of cluster evolu- tion. Industrial and Corporate Change, 19(1), 205-238.

McCormick, D. (2005). El futuro de los clusters y las cadenas productivas. Semestre Económico, 8(15), 87-102.

Ministerio de Agricultura y Desarrollo Rural (MADR) (2011). Informe de la Secretaría Técnica de la Cadena de Pasifloras. Bogotá: MADR.

Ministerio de Agricultura y Desarrollo Rural (MADR) (2012). Informe de la Secretaría Técnica de la Cadena de Pasifloras. Bogotá: MADR.

Pettigrew, A. M., Thomas, H., \& Whittington, R. (Eds.). (2010). Handbook of strategy and management. Los Ángeles - Londres - Nueva Delhi - Singapur - Washington: SAGE.

Porter, M. E. (1990). The competitive advantage of nations. Harvard Business Review, 68(2), 73-91.

Redman, J. M. (1994). Understanding state economics through industries studies. Washington: Council of Governor's Policy Advisors.

Rodríguez, F. B., \& Naranjo, J. C. (2008). La innovación tecnológica en el contexto de los clusters regionales. Cuadernos de Administración, 21(37), pp. 133-159.

Rosenfeld, S. (1995). Industrialstrength strategies: Regional business clusters and public policy. Washington: The Aspen Institute. 
Rosenfeld, S. A. (1997). Bringing business clusters into the mainstream of economic development. European Planning Studies, 5(1), 3-23.

Wroblewski, A., \& Leitner, A. (2009). The delphi method: Elicting ex- perts' knowledge in technology foresight. En A. Bogner, B. Littig \& W. Menz (Eds.), Interviewing experts (pp. 252-274). Basingstoke - Nueva York: Palgrave Macmillan. 